\title{
Does endocan level increase in women with polycystic ovary syndrome? A case - control study
}

\author{
Illhan Bahri Delibas ${ }^{1}$, Omer Erkan Yapca ${ }^{2}$, Esra Laloglu³ \\ ${ }^{1}$ Department of Obstetrics and Gynecology, Gaziosmanpasa University, Tokat, Turkey \\ ${ }^{2}$ Ataturk University, Department of Obstetrics and Gynecology, Erzurum, Turkey \\ ${ }^{3}$ Erzurum Public Health Laboratory, Erzurum, Turkey
}

\begin{abstract}
Objectives: To evaluate endocan levels of patients with polycystic ovary syndrome (PCOS) in comparison to healthy women.

Material and methods: A cross-sectional case-control study on 88 patients with PCOS (mean age, $22.06 \pm 4.24$ years; body mass index [BMI], $23.9 \pm 4.74 \mathrm{~kg} / \mathrm{m}^{2}$ ) and 87 age- and BMI-matched healthy women (mean age, $23.71 \pm 4.42$ years; BMl, $22.15 \pm 3.03 \mathrm{~kg} / \mathrm{m}^{2}$ ).

Results: Serum endocan level was significantly higher in PCOS group than control group $(540.9 \pm 280.3 \mathrm{pg} / \mathrm{mL}$ vs. $355.5 \pm 233.5 \mathrm{pg} / \mathrm{mL}$, respectively; $\mathrm{p}<0.001$ ). The presence of polycystic ovary finding on ultrasonography or oligomenorhea did not produce significant effect on serum endocan levels $(p>0.05)$. In PCOS group, endocan level was negatively correlated with BMI and C-reactive protein level, and positively correlated with high density lipoprotein level $(p<0.05)$.

Conclusions: Blood endocan level is increased in PCOS. Further studies are needed to evaluate the clinical value of blood endocan level as a marker for the risk of cardiovascular and metabolic diseases in patients with PCOS.
\end{abstract}

Key words: endocan, polycystic ovary syndrome, inflammation, case-control

Ginekologia Polska 2018; 89, 9: 500-505

\section{INTRODUCTION}

Polycystic ovary syndrome (PCOS) affects around $7 \%$ of women and is characterized by hyperandrogenism with or without oligo-anovulation [1]. The clinical signs and symptoms of PCOS change from puberty to perimenopausal period of patients. In addition to cosmetic complaints such as hirsutism and acne, patients with PCOS may have obesity, menstrual irregularity, and infertility. Increased levels of androgens in PCOS are associated with metabolic diseases including excess fat accumulation, obesity, and insulin resistance [1].

In patients with PCOS, the risk of cardiovascular and metabolic diseases, primarily diabetes, has increased [2-6]. For this reason, it is important not only to manage PCOS in terms of patient's specific complaints, but also to define potential risk of cardiovascular and metabolic diseases associated with this syndrome, and to inform patients about this risk. Previous studies have reported an inflammatory process underlying PCOS, which is associated with metabolic diseases, hyperandrogenism, insulin resistance, abdominal obesity, and endothelial damage [2-8]. Endothelium dysfunction, a chronic inflammatory process which is an early sign of atherosclerosis, has been demonstrated in studies on PCOS [8-10].

Endocan, endothelial cell-specific molecule (ESM-1), is a soluble $50 \mathrm{kDa}$ molecular weight proteoglycan expressed in vascular endothelium [11]. It has been reported that endocan levels increase in many cancers, sepsis, obesity, and a variety of inflammatory pathologies by playing a critical role in vascular injury in organ-specific pathologies and being a potential novel marker in endothelium-dependent pathologic disorders $[11,12]$. Although endothelial dysfunction in PCOS has been extensively studied, studies on the relationship between PCOS and endocan are limited $[13,14]$. 
Therefore, in this study, we aimed to evaluate endocan levels of patients with PCOS in comparison to healthy women in order to clarify the relationship between PCOS and endothelial injury.

\section{MATERIAL AND METHODS}

Study design and population

This was a cross-sectional case-control study conducted between January 2017 and April 2017 in the Department of Obstetrics and Gynecology, Gaziosmanpasa University Hospital, Tokat, Turkey. Eighty-eight patients with PCOS (mean age, $22.06 \pm 4.24$ years; body mass index [BMI], $23.9 \pm 4.74 \mathrm{~kg} / \mathrm{m}^{2}$ ) and 87 age- and BMl-matched subjects (mean age, $23.71 \pm 4.42$ years; $B M l, 22.15 \pm 3.03 \mathrm{~kg} / \mathrm{m}^{2}$ ) with normal menstrual cycles who presented to our outpatient clinic were included into the study. The selection criteria for the patients with PCOS were the absence of any significant abnormalities on physical examination except hirsutism; no lipid lowering, hypoglycemic, antihypertensive or hormone replacement therapy; normal thyroid function and prolactin level; absence of history or evidence of metabolic, cardiovascular, respiratory or hepatic disease. The diagnosis of PCOS was made in accordance with the criteria proposed at the Rotterdam revised consensus meeting (The Rotterdam European Society for Human Reproduction and Embryology [ESHRE]/American Society for Reproductive Medicine [ASRM]-Sponsored PCOS Consensus Workshop Group, 2004) [15]. All eligible patients met at least 2 of the 3 following criteria: [a] oligomenorrhea or amenorrhea, [b] clinical and/ or biochemical signs of hyperandrogenism (hirsutism and acne), and [c] polycystic ovaries. Subjects with possible ovarian tumors, congenital adrenal hyperplasia or BMI greater than $30 \mathrm{~kg} / \mathrm{m}^{2}$ were excluded from the study. All patients and control subjects were non-smoker, normotensive $(<$ $120 / 80 \mathrm{~mm} \mathrm{Hg}$ in two measurements), had regular daily activity and not regular consumer of alcoholic beverages. None of the subjects were pregnant during the study procedures.

The study was approved by the Clinical Research Ethics Committee of Gaziosmanpasa University School of Medicine (16-KAEK-038), and all patients and control subjects gave written informed consent before enrollment. The study was conducted according to the principles of the latest version of Helsinki Declaration.

\section{Study procedures}

All the participants were evaluated in one study visit, during which data on medical history, physical examination including anthropometric measurements, laboratory test, and pelvic ultrasonography (US) were recorded. BMI $\left(\mathrm{kg} / \mathrm{m}^{2}\right)$ was used as an estimate of overall adiposity in both groups. To evaluate the severity of hirsutism, hirsutism score, which includes clinical signs of hyperandrogenism was used.
A hirsutism score of more than 7 was considered as severe hirsutism [16]. The presence of PCO on US was recorded. All the measurements were made by the same physician.

For laboratory tests, venous blood samples were drawn on the third to fifth day of menstrual cycle between 08:00 and 10:00 a.m. after a 12-hour fasting. Glucose, total cholesterol, triglyceride, high-density lipoprotein (HDL)cholesterol, and C-reactive protein (CRP) levels were measured by enzymatic colorimetric method using Olympus AU 600 auto analyzer (Olympus Diagnostics, GmbH, Hamburg, Germany). Low-density lipoprotein (LDL)-cholesterol level was estimated by the Friedewald formula [LDL-cholester$\mathrm{ol}=$ (totalcholesterol) $-(\mathrm{HDL}$-cholesterol)-(triglyceride/5)]. Luteinizing hormone (LH), follicle stimulant hormone (FSH), estradiol (E2), total testosterone, dehydroepiandrosterone sulphate (DHEA-S), and insulin were measured in serum by chemiluminescence, using Modular E-170 Immunologic Analyzer (Roche Diagnostics, Osaka, Japan).

\section{Blood endocan level measurements}

The serum samples for endocan, i.e. ESM-1, were first stored for coagulation and then centrifuged for $15 \mathrm{~min}$ at $4000 \mathrm{xg}$ at $+4^{\circ} \mathrm{C}$. The serum samples obtained divided into aliquots and stored in a deep freeze at $-80^{\circ} \mathrm{C}$ until analysis. ESM-1 levels in serum samples were measured using ELISA with a "Human ESM-1 ELISA Kit" (Elabscience Biotechnology Co. Ltd., Lot: AK0016DEC07042, Wuhan, China). It uses a double-antibody sandwich enzyme-linked immunosorbent assay. Samples like serum and standards were pipetted into the 96 well microplate being coated on with a monoclonal antibody (also known as Capture Antibody that is specific for C terminal of human ESM-1), and incubated for $90 \mathrm{~min}$. ESM-1 present within a sample is bound by the Capture Antibody. After washing away of any unbound molecules, a secondary monoclonal antibody specific for $\mathrm{N}$ terminal of ESM-1 that has been biotinylated, was added to the wells to incubate for another 1 hour. After a washing step, streptavidin-HRP (biotin-binding protein conjugated with polymers of horseradish peroxidase) was added and allowed to incubate for $30 \mathrm{~min}$. Unbound material was washed away. Chromogen solution was added and incubated for $15 \mathrm{~min}$ (protected from light) for the conversion of the colorless solution to a blue solution, the intensity of which was proportional to the amount of ESM-1 in the sample. As an effect of the acidic stop solution, the color has become yellow. The colored reaction product was measured using an automated ELISA reader at $450 \mathrm{~nm}$. The results were expressed as picograms per milliliter $(\mathrm{pg} / \mathrm{mL})$. The performance characteristics of this kit from the manifacturer's sheet are given as: The measuring ranges, sensitivity, intra-assay precision and inter-assay precision of the endocan kit were $15.63 \mathrm{pg} / \mathrm{mL}$ to $1000 \mathrm{pg} / \mathrm{mL}$, 
$9.375 \mathrm{pg} / \mathrm{mL},<8$ and $<10 \%$, respectively. Values beneath the detection limit of $15.63 \mathrm{pg} / \mathrm{mL}$ were regarded as ' 0 '.

\section{Statistical analysis}

The power analysis was performed using G Power 3.1 for Windows (Heinrich-Heine-Universität Düsseldorf, Düsseldorf, Germany) [17]. Sample size calculation was based on the mean endocan levels. Mean endocan levels were reported as $621.82 \pm 345.41 \mathrm{pg} / \mathrm{mL}$ and $391.40 \pm 234.97 \mathrm{pg} / \mathrm{mL}$ in our pilot study. According to our pilot study results for circulating endocan levels, the minimum required size of the study population was calculated to be 70 subjects $(a=0.05$ and the study power $=0.90)$.

Quantative data were summarized using mean and standard deviation. Independent samples t test or one way analysis of variance (ANOVA) test were used to compare normal data between groups. For post-hoc comparisons between the pair-wise groups, the Tukey HSD test was used. Mann Whitney $U$ test was used to compare non-normal data between groups. Pearson correlation coefficients were used to test inter-variable relationships. A p value $<0.05$ was considered statistically significant. Analyses were performed using SPSS 19 (IBM SPSS Statistics 19, SPSS Inc., Somers, NY, USA).

\begin{tabular}{|c|c|c|c|}
\hline & $\begin{array}{l}\text { Control group } \\
(n=87)\end{array}$ & $\begin{array}{l}\text { PCOS patients } \\
(n=88)\end{array}$ & $\mathbf{p}$ \\
\hline \multicolumn{4}{|c|}{ Demographic and clinical findings } \\
\hline Age [years] & $23.71 \pm 4.42$ & $22.06 \pm 4.24$ & $0.012 a$ \\
\hline BMI [kg/m2] & $22.15 \pm 3.03$ & $23.9 \pm 4.74$ & $0.004 a$ \\
\hline Hirsutism score & $0.39 \pm 0.6$ & $9.95 \pm 4.23$ & $<0.001 \mathrm{~b}$ \\
\hline \multicolumn{4}{|c|}{ Hormonal parameters } \\
\hline FSH & $5.59 \pm 1.68$ & $5.54 \pm 1.9$ & $0.830 \mathrm{a}$ \\
\hline $\mathrm{LH}$ & $8.2 \pm 3.3$ & $10.95 \pm 7.47$ & $0.002 \mathrm{a}$ \\
\hline DHEA-S & $92.62 \pm 36.37$ & $240.36 \pm 122.01$ & $<0.001 \mathrm{~b}$ \\
\hline Total testosterone & $0.06 \pm 0.1$ & $0.56 \pm 0.28$ & $<0.001 b$ \\
\hline E2 & $59.99 \pm 15.79$ & $67.78 \pm 69.51$ & $0.037 b$ \\
\hline TSH & $1.68 \pm 0.58$ & $1.82 \pm 0.98$ & $0.252 a$ \\
\hline \multicolumn{4}{|c|}{ Biochemical parameters } \\
\hline Fasting blood glucose & $79.45 \pm 6.6$ & $88.66 \pm 9.57$ & $<0.001 a$ \\
\hline Triglyceride & $72.56 \pm 12.75$ & $110.28 \pm 50.79$ & $<0.001 a$ \\
\hline Total cholesterol & $106.3 \pm 16.35$ & $167.51 \pm 32.22$ & $0.001 a$ \\
\hline $\mathrm{HDL}$ & $53.44 \pm 3.66$ & $51.64 \pm 9.71$ & $0.107 a$ \\
\hline LDL & $90.13 \pm 9.43$ & $110.11 \pm 25.14$ & $<0.001 a$ \\
\hline CRP & $3.84 \pm 1.6$ & $5.33 \pm 4.72$ & $<0.001 b$ \\
\hline
\end{tabular}

Data are shown as mean \pm standard deviation; ${ }^{\mathrm{a}}$ Independent Samples t test; ${ }^{b}$ Mann Whitney U test; PCOS — polycystic ovary syndrome; BMI — body mass index; $\mathrm{FSH}$ - follicle stimulant hormone; $\mathrm{LH}$ - luteinizing hormone DHEA-S - luteinizing hormone; E2 - estradiol; TSH - thyroid stimulating hormone; HDL — high-density lipoprotein; LDL — low-density lipoprotein; CRP - C-reactive protein

\section{RESULTS \\ Clinical findings}

The mean age of all the particants was $22.88 \pm 4.40$ years, and BMI was $23.03 \pm 4.06 \mathrm{~kg} / \mathrm{m}^{2}$. The frequency of PCO finding on US, oligomenorrhea, and hyperandrogenism was 73 (83.9\%), 69 (78.4\%), and 68 (77.3\%), respectively. Age, $\mathrm{BMI}$, and hirsutism score of $\mathrm{PCOS}$ patients were significantly higher than control group ( $p<0.05$ ) (Tab. 1). According to $\mathrm{BMI}$, of all the study population, $32(18.3 \%)$ and $15(8.5 \%)$ were overweight $\left(25-29.9 \mathrm{~kg} / \mathrm{m}^{2}\right)$ and obese $\left(\geq 30 \mathrm{~kg} / \mathrm{m}^{2}\right)$, respectively. On the other hand, among PCOS patients, $20(22.7 \%)$ and $14(15.9 \%)$ were overweight and obese, respectively.

\section{Biochemical findings}

Biochemical and hormonal profiles of the control group were within normal ranges. Among biochemical parameters, triglyceride, total cholesterol, LDL, fasting blood glucose and CRP were significantly higher in PCOS group compared to control group $(p<0.05)$ (Tab. 1). For hormonal parameters, LH, E2, DHEA-S and total testosterone were significantly higher in PCOS group compared to control group $(p<0.05)$, as FSH and TSH were comparable between groups (Tab. 1).

\begin{tabular}{|c|c|c|c|c|}
\hline & & \multicolumn{3}{|c|}{ Endocan (pg/mL) } \\
\hline & & $\begin{array}{l}\text { Total } \\
(n=175)\end{array}$ & $\begin{array}{l}\text { PCOS group } \\
(n=88)\end{array}$ & $\begin{array}{l}\text { Control group } \\
(n=87)\end{array}$ \\
\hline \multirow{2}{*}{ BMI } & $r$ & -0.210 & -0.428 & -0.090 \\
\hline & $p$ & 0.005 & $<0.001$ & 0.406 \\
\hline \multirow{2}{*}{$\begin{array}{l}\text { Hirsutism } \\
\text { score }\end{array}$} & $r$ & 0.231 & -0.199 & 0.512 \\
\hline & $\mathrm{p}$ & 0.002 & 0.063 & $<0.001$ \\
\hline \multirow{2}{*}{$\mathrm{LH}$} & $r$ & 0.229 & 0.186 & 0.128 \\
\hline & $\mathrm{p}$ & 0.002 & 0.083 & 0.237 \\
\hline \multirow{2}{*}{ DHEA-S } & $r$ & 0.273 & 0.038 & 0.279 \\
\hline & $p$ & $<0.001$ & 0.723 & 0.009 \\
\hline \multirow{2}{*}{$\begin{array}{l}\text { Total } \\
\text { testosterone }\end{array}$} & $r$ & 0.346 & 0.057 & 0.453 \\
\hline & $\mathrm{p}$ & $<0.001$ & 0.597 & $<0.001$ \\
\hline \multirow{2}{*}{$\begin{array}{l}\text { Fasting blood } \\
\text { glucose }\end{array}$} & $r$ & 0.328 & -0.007 & 0.559 \\
\hline & $p$ & $<0.001$ & 0.947 & $<0.001$ \\
\hline \multirow{2}{*}{$\begin{array}{l}\text { Total } \\
\text { cholesterol }\end{array}$} & $r$ & 0.299 & -0.028 & 0.284 \\
\hline & $\mathrm{p}$ & $<0.001$ & 0.799 & 0.008 \\
\hline \multirow{2}{*}{$\mathrm{HDL}$} & $r$ & 0.158 & 0.392 & -0.309 \\
\hline & $\mathrm{p}$ & 0.037 & $<0.001$ & 0.004 \\
\hline \multirow{2}{*}{ CRP } & $r$ & -0.093 & -0.424 & 0.648 \\
\hline & $p$ & 0.222 & $<0.001$ & $<0.001$ \\
\hline
\end{tabular}

$r$ denotes Pearson correlation coefficient; PCOS - polycystic ovary syndrome; $\mathrm{BMI}$ - body mass index; LH: luteinizing hormone; DHEA-S — luteinizing hormone; $\mathrm{HDL}$ - high-density lipoprotein; CRP — C-reactive protein 


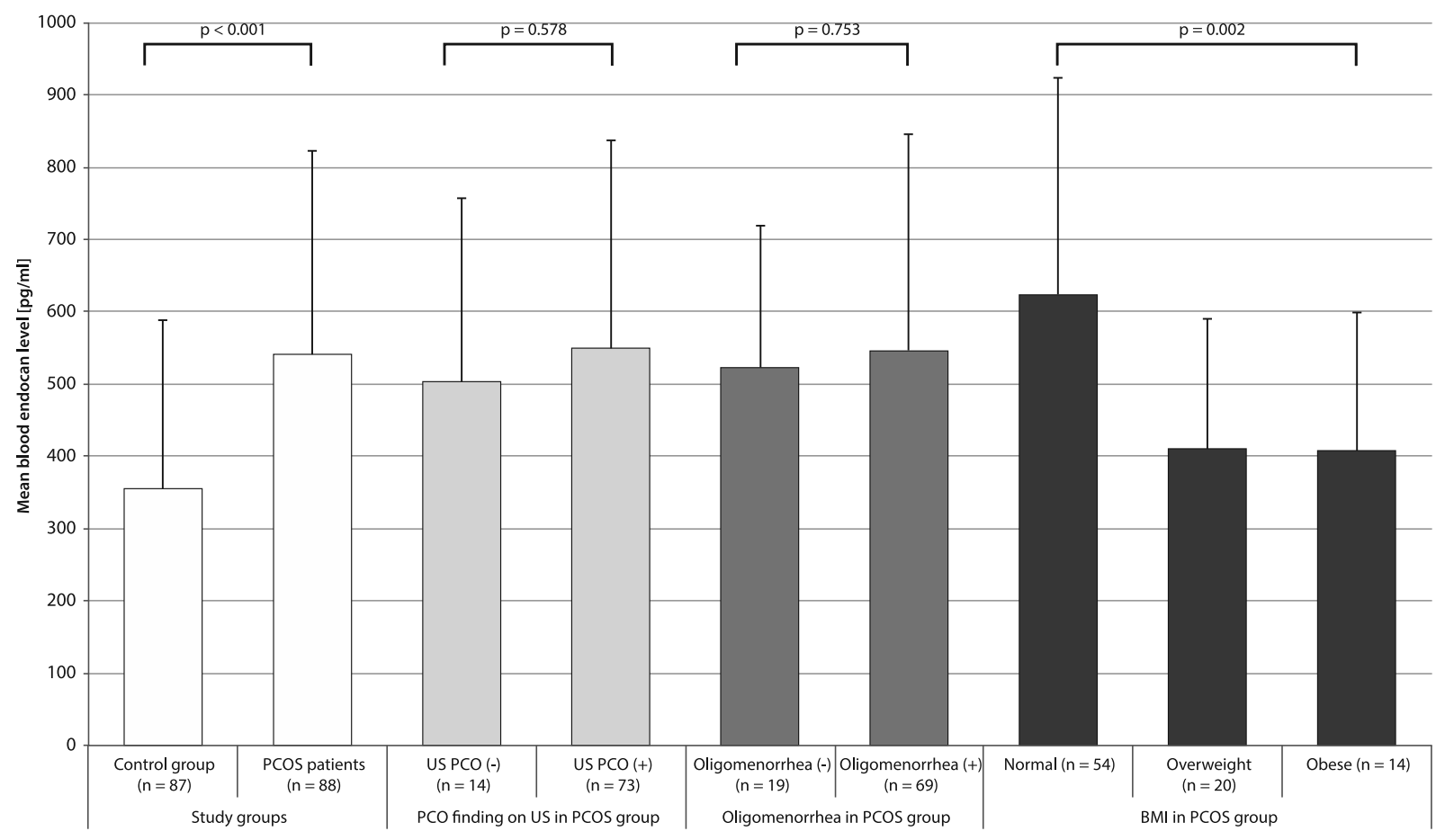

Figure 1. The mean blood endocan level in study groups and with respect to PCO finding on US, oligomenorrhea, and BMI in PCOS group. Vertical bars represent standard deviation. PCO — polycystic ovary; US — ultrasonography; BMI — body mass index; PCOS — polycystic ovary syndrome

\section{Serum endocan levels}

Serum endocan level was significantly higher in PCOS group than control group $(540.9 \pm 280.3 \mathrm{pg} / \mathrm{mL}$ vs. $355.5 \pm 233.5 \mathrm{pg} / \mathrm{mL}$, respectively; $\mathrm{p}<0.001$ ) (Fig. 1). The presence of PCO finding on US and oligomenorrhea in PCOS group did not produce significant effect on blood endocan levels ( $p>0.05$ ) (Fig. 1). However, overweight and obese PCOS patients had significantly lower blood endocan levels than normal weight PCOS patients $(p=0.002)$ (Fig. 1).

As BMI and $\mathrm{LH}$ were not correlated with endocan levels in control group; hirsutism score, DHEA-S, total testosterone, fasting blood glucose, total cholesterol, and CRP were positively and HDL was negatively correlated with endocan levels (Tab. 2). In PCOS group, there was a weak-to-moderate negative correlation between endocan levels and $\mathrm{BMI}$ and CRP, and positive correlation between endocan levels and HDL (Tab. 2).

\section{DISCUSSION}

In this case-control study, we primarily showed that serum endocan level is increased in patients with PCOS. Since BMI and CRP, the parameters related with inflammatory process, were negatively correlated with blood endocan level, hormonal rather than inflammatory/endothelial changes of PCOS are implicated for the increase in circulating endocan level. The presence of PCO finding on US or oligomenorrhea did not affect the endocan level, suggesting that there is no relation between imaging findings and menstrual symptoms of PCOS and endocan level.

Endocan is a circulating endothelium-derived proteoglycan. An increase in tissue or blood endocan level shows endothelial activation and neovascularization, which are indicators of inflammation and tumor progression [18]. Therefore, blood endocan level has been used as a biomarker for various cancers including ovarian or endometrial carcinoma, pre-eclampsia, and inflammation-related diseases such as cirrhosis, sepsis, hypertension, and Behcet's disease [18-24]. As well as presence and severity of inflammation-related diseases, these markers indicate cardiovascular and metabolic risk of patients [25]. Furthermore, some studies proposed endocan as a potential target for treatment of these diseases [26, 27].

It has been known that PCOS is a low-level chronic inflammation with increased serum level of inflammatory markers such as interleukin-1, interleukin-6, CRP, and tumor necrosis factor alpha $[28,29]$ Accordingly, in the present study biochemical and hormonal profiles of patients with PCOS showed significantly higher level of cardiovascular and inflamatory parameters regardless of PCO finding on US, oligomenorrhea, and BMI. The low-grade inflammation in PCOS is commonly associated with endothelial dysfunction regardless of $\mathrm{BMI}[30,31]$. Since increased level of circulating endocan is associated with endothelial dysfunction in 
inflammatory conditions, which was suggested as an early sign of atherogenesis, endocan may have a potential role as a prognostic biomarker in PCOS and indicator of cardiovascular risk [32, 33].

In a recent study, Bicer et al. reported an increased level of circulating endocan levels in women with PCOS compared with controls $(5.99 \pm 2.37 \mathrm{ng} / \mathrm{mL}$ vs. $3.66 \pm 1.79 \mathrm{ng} / \mathrm{mL}$, $\mathrm{p}<0.001$ ) [14]. Similarly, in our study, which is the second study in the literature evaluating endocan level in PCOS, serum endocan level was significantly higher in PCOS group than the control group $(540.9 \pm 280.3 \mathrm{pg} / \mathrm{mL}$ vs. $355.5 \pm 233.5 \mathrm{pg} / \mathrm{mL}$, respectively; $\mathrm{p}<0.001$ ).

Lean women populations with PCOS are a specific group and have different phenotypic, metabolic, and hematologic characteristics than obese women with PCOS and there is still an ongoing debate regarding this category of patients. It has been shown that obesity, and not PCOS status per se, was a major determinant of the circulating inflammatory markers TNF a, soluble type 2 TNF receptor, IL-6, and hs-CRP [34]. While the source of excess circulating TNF a in PCOS is likely to be adipose tissue in the obese but remains unknown in lean women with the disorder [35]. Therefore studies suggest that obesity is not independently associated with these markers of inflammation.

While they did not stratify PCOS subjects based on BMI, Bicer et al. also found that endocan is an independent predictor for carotid intima media thickness, which indicates risk of cardiovascular events, thus suggested that blood endocan level can be used to predict increased cardiovascular risk in PCOS patients [14]. However, in our study we interestingly found that overweight and obese PCOS patients had significantly lower serum endocan levels than normal weight PCOS patients, and in PCOS group, there was a weak-to-moderate negative correlation between endocan levels and BMI and CRP, and positive correlation between endocan levels and $\mathrm{HDL}$, indicating that endocan is not a direct marker of inflammation or cardiovascular risk in PCOS. We suggest that the increased endocan levels in lean PCOS patients compared to obese women with PCOS in our study may be explained by [1] the intracellular signalling pathways that govern the relationship between inflammatory response and circulating endocan levels may be more impaired in lean women with PCOS than obese women with PCOS or [2] endocan production may be regarded as part of a protective mechanism in the crosstalk of multiple signalling mediators, fighting with the state of increased inflammation. We think our study contributes to our understanding on the presence of PCOS in lean women while future studies in this field should define the Rotterdam phenotype of subjects, stratify subjects based on BMI, use clinically relevant cut offs rather than comparison of means and ultimately provide longitudinal data on changes in biomarkers and their correlation with metabolic and clinical manifestations of PCOS.

Furthermore, in our study the presence of PCO finding on ultrasonography and oligomenorrhea in PCOS group did not produce significant effect on serum endocan levels. Therefore, we suggest that there is no relation between symptoms of PCOS and endocan level. On the other hand, in control group of women with normal menstrual cyles; hirsutism score, DHEA-S, total testosterone, fasting blood glucose, total cholesterol, and CRP were positively and HDL was negatively correlated with endocan levels.

The main limitation of our study was its cross-sectional design, which limits the interpretation of cause-effect relation of increased level of circulating endocan level and PCOS. Nevertheless, this is among the few studies on the blood endocan level in patients with PCOS. Further studies are needed to clarify the exact mechanism underlying the increase in blood endocan level in PCOS.

\section{CONCLUSIONS}

Serum endocan level is increased in patients with PCOS, which seems to result from hormonal or other factors which need to be evaluated rather than inflammatory/endothelial changes of PCOS. Further studies are needed to conclude on mechanism of increased endocan level in PCOS, and to evaluate the clinical value of endocan as a biomarker for inflammation and cardiovascular risk, and potential therapeutic target in PCOS.

\section{Acknowledgements}

This study was funded by the Gaziosmanpasa University Scientific Research Funding (no: 2016/14) and registered at clinicaltrials.gov (identifier NCT03019679).

\section{Conflict of interest}

Authors declare no conflicts of interest.

\section{REFERENCES}

1. Carmina E, Orio F, Palomba S, et al. Endothelial Dysfunction in PCOS: Role of Obesity and Adipose Hormones. The American Journal of Medicine. 2006; 119(4): 356.e1-356.e6, doi: 10.1016/j.amjmed.2005.10.059.

2. Ehrmann DA, Barnes RB, Rosenfield RL, et al. Prevalence of impaired glucose tolerance and diabetes in women with polycystic ovary syndrome. Diabetes Care. 1999; 22(1): 141-146, indexed in Pubmed: 10333916.

3. Legro RS, Kunselman AR, Dodson WC, et al. Prevalence and predictors of risk for type 2 diabetes mellitus and impaired glucose tolerance in polycystic ovary syndrome: a prospective, controlled study in 254 affected women. J Clin Endocrinol Metab. 1999; 84(1): 165-169, doi: 10.1210/jcem.84.1.5393, indexed in Pubmed: 9920077.

4. Paradisi G, Steinberg HO, Hempfling A, et al. Polycystic ovary syndrome is associated with endothelial dysfunction. Circulation. 2001; 103(10): 1410-1415, indexed in Pubmed: 11245645.

5. Diamanti-Kandarakis E, Alexandraki K, Protogerou A, et al. Metformin administration improves endothelial function in women with polycystic ovary syndrome. Eur J Endocrinol. 2005; 152(5): 749-756, doi: 10.1530/eje.1.01910, indexed in Pubmed: 15879361.

6. Diamanti-Kandarakis E, Spina G, Kouli C, et al. Increased endothelin-1 levels in women with polycystic ovary syndrome and the beneficial effect 
of metformin therapy. J Clin Endocrinol Metab. 2001;86(10): 4666-4673, doi: 10.1210/jcem.86.10.7904, indexed in Pubmed: 11600523.

7. Kelly CC, Lyall $\mathrm{H}$, Petrie JR, et al. Low grade chronic inflammation in women with polycystic ovarian syndrome. J Clin Endocrinol Metab. 2001; 86(6): 2453-2455, doi: 10.1210/jcem.86.6.7580, indexed in Pubmed: 11397838.

8. Diamanti-Kandarakis E, Paterakis T, Alexandraki K, et al. Indices of low-grade chronic inflammation in polycystic ovary syndrome and the beneficial effect of metformin. Hum Reprod. 2006; 21(6): 1426-1431, doi: 10.1093/humrep/del003, indexed in Pubmed: 16497699.

9. Shimokawa H. Primary endothelial dysfunction: atherosclerosis. J Mol Cell Cardiol. 1999; 31(1): 23-37, doi: 10.1006/jmcc.1998.0841, indexed in Pubmed: 10072713.

10. Ross R. Atherosclerosis--an inflammatory disease. N Engl J Med. 1999; 340(2): 115-126, doi: 10.1056/NEJM199901143400207, indexed in Pubmed: 9887164.

11. Aparci M, Isilak Z, Uz O, et al. Endocan and endothelial dysfunction. Angiology. 2015; 66(5): 488-489, doi: 10.1177/0003319714568791, indexed in Pubmed: 25632053.

12. Mihajlovic DM, Lendak DF, Brkic SV, et al. Endocan is useful biomarker of survival and severity in sepsis. Microvasc Res. 2014; 93: 92-97, doi: 10.1016/j.mvr.2014.04.004, indexed in Pubmed: 24769132.

13. Kao YH, Chiu WC, Hsu Ml, et al. Endothelial progenitor cell dysfunction in polycystic ovary syndrome: implications fort he genesis of cardiovascular diseases. Int J Fert Steril. 2013; 6: 208-213.

14. Bicer M, Guler A, Unal Kocabas G, et al. Endocan is a predictor of increased cardiovascular risk in women with polycystic ovary syndrome. Endocr Res. 2017; 42(2): 145-153, doi: 10.1080/07435800.2016.1255896, indexed in Pubmed: 27911121.

15. Rotterdam ESHRE/ASRM-Sponsored PCOS Consensus Workshop Group, Rotterdam ESHRE/ASRM-Sponsored PCOS consensus workshop group. Revised 2003 consensus on diagnostic criteria and long-term health risks related to polycystic ovary syndrome (PCOS). Hum Reprod. 2004; 19(1): 41-47, indexed in Pubmed: 14688154.

16. FERRIMAN D, GALLWEY JD. Clinical assessment of body hair growth in women. J Clin Endocrinol Metab. 1961; 21: 1440-1447, doi: 10.1210/jcem-21-11-1440, indexed in Pubmed: 13892577.

17. Faul F, Erdfelder E, Buchner A, et al. Statistical power analyses using G*Power 3.1: tests for correlation and regression analyses. Behav Res Methods. 2009; 41(4): 1149-1160, doi: 10.3758/BRM.41.4.1149, indexed in Pubmed: 19897823.

18. Kali A, Shetty KS. Endocan: a novel circulating proteoglycan. Indian J Pharmacol. 2014; 46(6): 579-583, doi: 10.4103/0253-7613.144891, indexed in Pubmed: 25538326.

19. Balta I, Balta S, Koryurek OM, et al. Serum endocan levels as a marker of disease activity in patients with Behçet disease. J Am Acad Dermatol. 2014; 70(2): 291-296, doi: 10.1016/j.jaad.2013.09.013, indexed in Pubmed: 24176522.

20. Saldir M, Tunc T, Cekmez F, et al. Endocan and Soluble Triggering Receptor Expressed on Myeloid Cells-1 as Novel Markers for Neonatal Sepsis. Pediatr Neonatol. 2015; 56(6): 415-421, doi: 10.1016/j.pedneo.2015.03.006, indexed in Pubmed: 26341458.

21. Celık T, Balta S, Karaman M, et al. Endocan, a novel marker of endothelial dysfunction in patients with essential hypertension: comparative effects of amlodipine and valsartan. Blood Press. 2015; 24(1): 55-60, doi: 10.3109/08037051.2014.972816, indexed in Pubmed: 25390761.

22. Zuwala-Jagiello J, Simon K, Kukla M, et al. Increased circulating endocan in patients with cirrhosis: relation to bacterial infection and severity of disease. J Physiol Pharmacol. 2017; 68(2): 273-282, indexed in Pubmed: 28614777.

23. Laloglu E, Kumtepe $Y$, Aksoy H, et al. Serum endocan levels in endometrial and ovarian cancers. J Clin Lab Anal. 2017; 31(5), doi: 10.1002/jcla.22079, indexed in Pubmed: 27734523.

24. Schuitemaker JHN, Cremers TI, Van Pampus MG, et al. Changes in endothelial cell specific molecule 1 plasma levels during preeclamptic pregnancies compared to healthy pregnancies. Pregnancy Hypertens. 2018; 12: 58-64, doi: 10.1016/j.preghy.2018.02.012, indexed in Pubmed: 29674200.

25. Samy N, Hashim M, Sayed M, et al. Clinical significance of inflammatory markers in polycystic ovary syndrome: their relationship to insulin resistance and body mass index. Dis Markers. 2009; 26(4): 163-170, doi: 10.3233/DMA-2009-0627, indexed in Pubmed: 19729797.

26. Sarrazin $S$, Adam E, Lyon M, et al. Endocan or endothelial cell specific molecule-1 (ESM-1): a potential novel endothelial cell marker and a new target for cancer therapy. Biochim Biophys Acta. 2006; 1765(1): 25-37, doi: 10.1016/j.bbcan.2005.08.004, indexed in Pubmed: 16168566.

27. LeeW, Ku SK, Kim SW, et al. Endocan elicits severe vascular inflammatory responses in vitro and in vivo. J Cell Physiol. 2014; 229(5): 620-630, doi: 10.1002/jcp.24485, indexed in Pubmed: 24446198.

28. Zafari Zangeneh F, Naghizadeh MM, Masoumi M. Polycystic ovary syndrome and circulating inflammatory markers. Int J Reprod Biomed (Yazd). 2017; 15(6): 375-382, indexed in Pubmed: 29177240

29. Escobar-Morreale HF, Luque-Ramírez M, González F. Circulating inflammatory markers in polycystic ovary syndrome: a systematic review and metaanalysis. Fertil Steril. 2011; 95(3): 1048-10458.e1, doi: 10.1016/j. fertnstert.2010.11.036, indexed in Pubmed: 21168133.

30. Rajendran S, Willoughby SR, Chan WP, et al. Polycystic ovary syndrome is associated with severe platelet and endothelial dysfunction in both obese and lean subjects. Atherosclerosis. 2009; 204(2): 509-514, doi: 10.1016/j.atherosclerosis.2008.09.010, indexed in Pubmed: 19027116.

31. Repaci A, Gambineri A, Pasquali R. The role of low-grade inflammation in the polycystic ovary syndrome. Mol Cell Endocrinol. 2011; 335(1): 30-41, doi: 10.1016/j.mce.2010.08.002, indexed in Pubmed: 20708064.

32. Balta S, Mikhailidis DP, Demirkol S, et al. Endocan: A novel inflammatory indicator in cardiovascular disease? Atherosclerosis. 2015; 243(1): 339-343, doi: 10.1016/j.atherosclerosis.2015.09.030, indexed in Pubmed: 26448266.

33. Cox LAE, van Eijk LT, Ramakers BPC, et al. Inflammation-induced increases in plasma endocan levels are associated with endothelial dysfunction in humans in vivo. Shock. 2015; 43(4): 322-326, doi: 10.1097/SHK.0000000000000320, indexed in Pubmed: 25565643.

34. Mohlig $M$, Spranger J, Osterhoff $M$, et al. The polycystic ovary syndrome per se is not associated with increased chronic inflammation. European Journal of Endocrinology. 2004; 150(4): 525-532, doi: 10.1530/eje.0.1500525.

35. Hotamisligil GS, Shargill NS, Spiegelman BM. Adipose expression of tumor necrosis factor-alpha: direct role in obesity-linked insulin resistance. Science. 1993; 259(5091): 87-91, indexed in Pubmed: 7678183. 